\title{
A 2-Stage Implicit Runge-Kutta Method Based on Heronian Mean for Solving Ordinary Differential Equations
}

\author{
Adegoke Stephen Olaniyan ${ }^{1}$, Omolara Fatimah Bakre ${ }^{2}$, Moses Adebowale Akanbi ${ }^{1}$ \\ ${ }^{1}$ Department of Mathematics, Lagos State University, Lagos, Nigeria \\ ${ }^{2}$ Department of Mathematics, Federal College of Education (Technical), Lagos, Nigeria
}

Email address:

gokayola@gmail.com (A. S. Olaniyan), larabakre@gmail.com (O. F. Bakre), moses.akanbi@lasu.edu.ng (M. A. Akanbi)

\section{To cite this article:}

Adegoke Stephen Olaniyan, Omolara Fatimah Bakre, Moses Adebowale Akanbi. A 2-Stage Implicit Runge-Kutta Method Based on Heronian Mean for Solving Ordinary Differential Equations. Pure and Applied Mathematics Journal. Vol. 9, No. 5, 2020, pp. 84-90.

doi: 10.11648/j.pamj.20200905.11

Received: July 23, 2020; Accepted: August 17, 2020; Published: September 8, 2020

\begin{abstract}
In recent times, the use of different types of mean in the derivation of explicit Runge-Kutta methods had been on increase. Researchers have explored explicit Runge-Kutta methods derivation by using different types of mean such as geometric mean, harmonic mean, contra-harmonic mean, heronian mean to name but a few; as against the conventional explicit Runge-Kutta methods which was viewed as arithmetic mean. However, despite efforts to improve the derivation of explicit Runge-Kutta methods with use of other types of mean, none has deemed it fit to extend this notion to implicit Runge-Kutta methods. In this article, we present the use of heronian mean as a basis for the construction of implicit Runge-Kutta method in a way of improving the conventional method which is arithmetic mean based. Numerical results was conducted on ordinary differential equations which was compared with the conventional two-stage fourth order implicit Runge-Kutta (IRK4) method and two-stage third order diagonally implicit Runge-Kutta (DIRK3) method. The results presented confirmed that the new scheme performs better than these numerical methods. A better Qualitative properties using Dalquist test equation were established.
\end{abstract}

Keywords: Implicit Runge-Kutta, Heronian Mean, Absolute Stability, Convergence, Ordinary Differential Equation

\section{Introduction}

Runge-Kutta methods are among the oldest and best understood methods in numerical analysis which have prompted researchers to explore them. Accordingly, Runge-Kutta methods represent an important family of implicit and explicit iterative methods for the approximation of ordinary differential equation in numerical analysis. However, due to the complexity of some real life mathematical models, explicit Runge-Kutta methods have been considered not stable enough due to its small region of absolute stability. Nevertheless, much work have been done recently in the formulation of explicit Runge-Kutta based on averages (such as geometric mean, harmonic mean, heronian mean, contra-harmonic mean among others) other than the conventional arithmetic mean for the numerical solution of ordinary differential equation.

In 1995, Evans D. J and Yaacob N. B. [11] developed a new 4th order Runge-Kutta method based on the contra-harmonic mean. They asserted that it is accurate compared to its equivalents. Also in Evans D. J. and Yaacob N. B. [10], a new 4th Order Runge-Kutta method based on the heronian formula was presented where heronian mean was used in the construction. The method was compared with several Runge-Kutta methods of 4th order based on variety of mean. Ponalagusamy P. J. A. and Chandra M. (2011) [17] developed a new Fifth-Order Fifth Stage Runge-Kutta method on heronian mean wherein heronian mean was used in the construction of 5th stage explicit Runge-Kutta methods. They confirmed that their scheme compete favorably well with 5th stage explicit Runge-Kutta method. A new 4th order embedded method based on the harmonic mean was constructed by Yaacob N. and Sanugi B. (1998) [20] in which harmonic mean was embedded in the arithmetic mean viewed Runge-kutta methods. The proposed method was found accurate and cost effective compared to the classical methods. On 3-stage geometric explicit Runge-Kutta methods for singular autonomous IVP in ordinary differential equations 
was developed by Akanbi (2010) [5]. In this paper, geometric mean was incorporated in the classical 3-stage Runge-Kutta methods. The scheme showed that it is stable, efficient and accurate when compared with some other conventional methods. A third order harmonic mean for autonomous IVP was constructed by Wusu A. S. et al. (2010) [19]. The method was derived based on harmonic mean and was confirmed to be better than any third order of any form of explicit Runge-Kutta methods. Wusu A. S. et al. (2015) [18] went further to introduce the harmonic mean as against the usual arithmetic average in standard 4th-Order Runge-Kutta scheme and compared their scheme with 4-Stage 4th-Order Runge-Kutta, 4th-Order Runge-Kutta method based on heronian mean formula and 4th-Order Runge-Kutta based on harmonic mean by Evans D. J. and Yaacob N. B. [11]. They ascertained that their scheme was better than the above mentioned schemes.

Of recent, there had not been any significant effort to extend this notion to implicit Runge-Kutta though some interesting research on implicit Runge-Kutta methods had been forthcoming (see $[1-4,7,8,12,15,16]$ ). However, it is pertinent to extend this idea to implicit Runge-Kutta. To this end, a modest effort has been made in this paper to introduce heronian mean in the derivation of the classical implicit Runge-Kutta method. It has been discovered that the proposed scheme compete favorably than the classical implicit Runge Kutta and other implicit numerical methods. The convergence and stability analysis were discussed.

\section{Materials and Methods}

Consider the initial value problem (IVP) of an ordinary differential equation:

$$
\mathrm{y}^{\prime}=\mathrm{f}(\mathrm{y}(\mathrm{x})), \mathrm{y}\left(\mathrm{x}_{0}\right)=\mathrm{y}_{0}
$$

The proposed heronian implicit Runge-Kutta method to solve the IVP (1) is borne out of classical implicit Runge-Kutta methods whose derivation is based on arithmetic mean.

The general R-stage classical implicit Runge-Kutta method is defined by:

$$
\begin{gathered}
y_{n+1}-y_{n}=h \varphi\left(x_{n}, y_{n}, h\right) \\
\varphi\left(x_{n}, y_{n}, h\right)=\sum_{r=1}^{R} c_{r} k_{r} \\
k_{r}=f\left(x+h a_{r}, y+h \sum_{s=1}^{R} b_{r s} k_{s}\right)
\end{gathered}
$$

Where $a_{r}=\sum_{s=1}^{R} b_{r s}, r=1,2, \ldots, R$

The 2-stage implicit Runge-Kutta method to solve the IVP (1) is defined as

$$
\begin{gathered}
y_{n+1}=y_{n}=\frac{h}{2}\left(k_{1}+k_{2}\right) \\
k_{1}=f\left(x_{n}+\left(\frac{1}{2}+\frac{\sqrt{3}}{6}\right) h, y_{n}+\frac{1}{4} h k_{1}+\left(\frac{1}{4}+\frac{\sqrt{3}}{6}\right) h k_{2}\right) \\
k_{2}=f\left(x_{n}+\left(\frac{1}{2}-\frac{\sqrt{3}}{6}\right) h, y_{n}+\left(\frac{1}{4}-\frac{\sqrt{3}}{6}\right) h k_{1}-\frac{1}{4} h k_{2}\right)
\end{gathered}
$$

a method originally proposed by Hammer and Hollingsworth in Lambert [14] which was derived based on arithmetic mean. In this paper we are proposing a 2-stage heronian implicit Runge-Kutta method for autonomous differential equation given as

$$
y_{n+1}-y_{n}=h \varphi_{H}\left(y_{n}: h\right)
$$

where

$$
\begin{gathered}
\varphi_{H}\left(y_{n}: h\right)=c_{1}\left(\frac{k_{1}+k_{2}+\sqrt{k_{1} * k_{2}}}{3}\right) \\
k_{r}=f\left(y+h \sum_{s=1}^{R} b_{r s} k_{s}\right)
\end{gathered}
$$

For $\mathrm{R}=2$ and $\mathrm{r}=1,2 ;(4)$ becomes

$$
k_{r}=f\left(y+b_{r 1} k_{1} h+b_{r 2} k_{2} h\right)
$$

Expanding (5), we have

$$
k_{r}=f+h f_{y}\left(b_{r 1} k_{1}+b_{r 2} k_{2}\right)+\frac{h^{2}}{2} f_{y y}\left(b_{r 1} k_{1}+b_{r 2} k_{2}\right)^{2}+\frac{h^{3}}{6} f_{y y y}\left(b_{r 1} k_{1}+b_{r 2} k_{2}\right)^{3}+0\left(h^{4}\right)
$$

We will assume $\mathrm{k}_{1}$ and $\mathrm{k}_{2}$ in the form

$$
k_{r}=A_{r}+h B_{r}+h^{2} C_{r}+h^{3} D_{r}+0\left(h^{4}\right)
$$

Substituting for $k_{r}$ by (7) in (6), we have

$$
\begin{aligned}
A_{r}+h B_{r}+h^{2} C_{r}+h^{3} D_{r}=f+ & h f_{y}\left[b_{r 1}\left(A_{1}+h B_{1}+h^{2} C_{1}\right)+b_{2}\left(A_{2}+h B_{2}+h^{2} C_{2}\right)\right] \\
& +\frac{h^{2}}{2} f_{y y}\left[b_{r 1}\left(A_{1}+h B_{1}\right)+b_{r 2}\left(A_{2}+h B_{2}\right)\right]^{2}+\frac{h^{3}}{6} f_{y y y}\left[b_{r 1} A_{1}+b_{r 2} A_{2}\right]^{3}+0\left(h^{4}\right)
\end{aligned}
$$

Equating powers of $h$

$$
\begin{gathered}
A_{r}=f \\
B_{r}=a_{r} f_{y y}
\end{gathered}
$$




$$
\begin{gathered}
C_{r}=\left(b_{r 1} a_{1}+b_{r 2} a_{2}\right) f_{y}^{2}+\frac{1}{2} a_{r}^{2} f^{2} f_{y y} \\
D_{r}=\left[b_{r 1}\left(b_{11} a_{1}+b_{12} a_{2}\right)+b_{r 2}\left(b_{21} a_{1}+b_{22} a_{2}\right)\right] f f_{y}^{3}+\frac{1}{2} a_{r}^{2}\left(a_{1}^{2} b_{r 1}+a_{2}^{2} b_{r 2}\right) f^{2} f_{y y}+a_{r}\left(b_{r 1} a_{1}+b_{r 2} a_{2}\right) f^{2} f_{y} f_{y y}+\frac{1}{6} a_{r}^{3} f^{2} f_{y y y}
\end{gathered}
$$

From (3)

$$
k_{1} k_{2}=A_{1} A_{2}+A_{1} B_{2}+A_{2} B_{1}+B_{1} B_{2}+A_{2} C_{1}+B_{2} C_{1}+A_{1} C_{2}+B_{1} C_{2}+A_{2} D_{1}+A_{1} D_{2}+0\left(h^{4}\right)
$$

Substituting (8)-(11) in (12), simplifying and collecting like terms, we have

$$
\begin{aligned}
k_{1} k_{2}= & f^{2}\left[1+h\left(a_{1}+a_{2}\right) f_{y}+h^{2}\left(a_{1}\left(b_{21}+b_{11}\right)+a_{2}\left(b_{22}+b_{12}\right)+a_{1} a_{2}\right) f_{y}^{2}+\frac{h^{2}}{2}\left(a_{1}^{2}+a_{2}^{2}\right) f f_{y y}+\right. \\
& h^{3}\left(\left(b_{11} a_{1}+b_{12} a_{2}\right)\left(b_{21}+b_{11}+a_{2}\right)+\left(b_{21} a_{1}+b_{22} a_{2}\right)\left(b_{22}+b_{12}+a_{1}\right)\right) f_{y}^{3}+\frac{h^{3}}{2}\left(a_{1}^{2}\left(b_{21}+b_{11}+a_{2}\right)\right. \\
& \left.\left.+a_{2}^{2}\left(b_{22}+b_{12}+a_{1}\right)+2 a_{2}\left(b_{21} a_{1}+b_{22} a_{2}\right)+2 a_{1}\left(b_{11} a_{1}+b_{12} a_{2}\right)\right) f f_{y} f_{y y}+\frac{h^{3}}{2}\left(a_{1}^{3}+a_{2}^{3}\right) f^{2} f_{y y y}\right]+0\left(h^{4}\right)
\end{aligned}
$$

Using $(1+x)^{n}=1+\frac{n x}{1 !}+\frac{n(n-1) x^{2}}{2 !}+\ldots$

$$
\begin{aligned}
\sqrt{k_{1} k_{2}}= & f+\frac{h}{2}\left(a_{1}+a_{2}\right) f f_{y}+\frac{h^{2}}{2}\left(a_{1}\left(b_{21}+b_{11}\right)+a_{2}\left(b_{22}+b_{12}\right)+a_{1} a_{2} f f_{y}^{2}+\frac{h^{2}}{4}\left(a_{1}^{2}+a_{2}^{2}\right) f^{2} f_{y y}\right. \\
& +\frac{h^{3}}{2}\left(\left(b_{11} a_{1}+b_{12} a_{2}\right)\left(b_{21}+b_{11}+a_{2}\right)+\left(b_{21} a_{1}+b_{22} a_{2}\right)\left(b_{22}+b_{12}+a_{1}\right)\right) f f_{y}^{3}+\frac{h^{3}}{4}\left(a_{1}^{2}\left(b_{21}+b_{11}+a_{2}\right)\right. \\
& \left.+a_{2}^{2}\left(b_{22}+b_{12}+a_{1}\right)+2 a_{2}\left(b_{21} a_{1}+b_{22} a_{2}\right)+2 a_{1}\left(b_{11} a_{1}+b_{12} a_{2}\right)\right) f^{2} f_{y} f_{y y}+\frac{h^{3}}{12}\left(a_{1}^{3}+a_{2}^{3}\right) f^{2} f_{y y y} \\
& +\frac{h^{2}}{8}\left(a_{1}+a_{2}\right) f f_{y}^{2}-\frac{h^{3}}{4}\left(a_{1}+a_{2}\right)\left(a_{1}\left(b_{21}+b_{11}\right)+a_{2}\left(b_{22}+b_{12}+a_{1} a_{2}\right)\right) f f_{y}^{3} \\
& -\frac{h^{3}}{8}\left(a_{1}+a_{2}\right)\left(a_{1}^{2}+a_{2}^{2}\right) f^{2} f_{y} f_{y y}+\frac{h^{3}}{16}\left(a_{1}+a_{2}\right)^{3} f^{3} f_{y y y}+0\left(h^{4}\right)
\end{aligned}
$$

Collecting like terms and simplifying (3), (2) becomes

$$
\begin{aligned}
y_{n+1}-y_{n}= & h c_{1} f+\frac{h}{2} c_{1}\left(a_{1}+a_{2}\right) f f_{y}+\frac{h^{2}}{24} c_{1}\left[2 a_{1} a_{2}+12 a_{1}\left(b_{21}+b_{11}\right)-a_{1}^{2}+12 a_{2}\left(b_{22}+b_{12}\right)-a_{2}^{2}\right] f f_{y}^{2} \\
& +\frac{h^{2}}{4} c_{1}\left(a_{1}^{2}+a_{2}^{2}\right) f^{2} f_{y y}+\frac{h^{3}}{48} c_{1}\left[\left(2 a_{1}^{2} a_{2}+12 a_{1}^{2}\left(b_{21}+3 b_{11}\right)\right) f^{2} f_{y} f_{y y}-a_{1}^{2}\left(a_{2}+4 b_{11}-4 b_{21}\right) f f_{y}^{3}\right. \\
& +a_{1}\left(4 a_{2}\left(b_{11}-b_{12}-b_{21}+b_{22}\right)-a_{2}^{2}+24\left(b_{11}^{2}+b_{11} b_{21}+b_{21}\left(b_{12}+b_{22}\right)\right)\right) f f_{y}^{3}+2 a_{1} a_{2}\left(a_{2}+12\left(b_{12}+b_{21}\right)\right) f^{2} f_{y} f_{y y} \\
& +a_{2}\left(a_{2}^{2}+4 a^{2}\left(b_{12}-b_{22}\right)+24\left(b_{12}\left(b_{11}+b_{21}\right)+b_{12} b_{22}+b_{22}^{2}\right)\right) f f_{y}^{3}-2 a_{2}^{2}\left(a_{2}-6\left(b_{12}+3 b_{22}\right)\right) f^{2} f_{y} f_{y y} \\
& \left.+4 a_{2}^{3} f^{3} f_{y y y}+a_{1}^{3}\left(f f_{y}^{3}-2 f^{2} f_{y} f_{y y}+4 f^{3} f_{y y y}\right)\right]+O\left(h^{4}\right)
\end{aligned}
$$

Comparing the coefficients of $h$ in (15) with the Taylor's series expansion involving only partial derivatives with respect to y of the form:

$$
\begin{aligned}
\Phi_{T}(x, y, h)=f & \left.+\frac{h}{2 !} f f_{y}+\frac{h^{2}}{3 !} f f_{y}^{2}+f^{2} f_{y y}\right)+\frac{h^{3}}{4 !}\left(4 f^{2} f_{y} f_{y y}+f f_{y}^{3}+f^{3} f_{y y y}\right) \\
& +\frac{h^{4}}{5 !}\left(7 f^{3} f_{y} f_{y y y}+4 f^{3} f_{y y}^{2}+11 f^{2} f_{y}^{2} f_{y y}+f f_{y}^{4}+f^{4} f_{y y y y}\right)
\end{aligned}
$$

we have the following set of equations:

$$
\begin{aligned}
& c_{1}=1 \\
& c_{1}\left(a_{1}+a_{2}\right)=1 \\
& \frac{c_{1}}{4}\left[-a_{1}^{2}+2 a_{1}\left(a_{2}+6\left(b_{21}+b_{11}\right)\right)+a_{2}\left(-a_{2}+12\left(b_{22}+b_{12}\right)\right]=1\right. \\
& \frac{c_{1}}{4}\left(a_{1}^{2}+a_{2}^{2}\right)=\frac{1}{6} \\
& \frac{c_{1}}{48}\left[-a_{1}^{2}\left(a_{2}+4 b_{11}-4 b_{21}\right)+a_{1}\left(a_{2}^{2}+4 a_{2}\left(b_{11}-b_{12}-b_{21}+\right.\right.\right.
\end{aligned}
$$




$$
\begin{aligned}
& \left.\left.\left.b_{22}\right)+24\left(b_{11}^{2}+b_{11} b_{21}+b_{21}\left(b_{12}+b_{22}\right)\right)\right)+a_{2}\left(a_{2}^{2}+4 a_{1}\left(b_{12}-b_{22}\right)+24\left(b_{12}\left(b_{11}+b_{21}\right)+b_{12} b_{22}+b_{22}^{2}\right)\right)+a_{1}^{3}\right]=\frac{1}{24} \\
& \frac{c_{1}}{48}\left[2 a_{1}^{2}\left(a_{2}+6\left(b_{21}+3 b_{11}\right)\right)+2 a_{1} a_{2}\left(a_{2}+12\left(b_{12}+b_{21}\right)-2 a_{2}^{2}\left(a_{2}-6\left(b_{12}+3 b_{22}\right)\right)-2 a_{1}^{3}\right)\right]=\frac{1}{6} \\
& c_{1}\left(a_{1}^{3}+a_{2}^{3}\right)=\frac{1}{2}
\end{aligned}
$$

Which were solved to obtain a 2-Stage HIRK scheme stated below:

$$
\begin{aligned}
& y_{n+1}=y_{n}+h \frac{k_{1}+k_{2}+\sqrt{k_{1} k_{2}}}{3} \\
& k_{1}=f\left(y+0.265364 k_{1} h+0.598226 k_{2} h\right) \\
& k_{2}=f\left(y-0.0333476 k_{1} h+0.244672 k_{2} h\right)
\end{aligned}
$$

\section{Stability and Convergence of the Scheme}

Analysis of the properties of a numerical method is best done using a standard linear test problem $\mathrm{y}^{\prime}=\lambda \mathrm{y}$ proposed by Dalquist where the solution is $y=e^{\lambda y}$ and $\lambda$ is a parameter [14].

\subsection{Absolute Stability of the Scheme}

The traditional criterion for ensuring that a numerical method is stable is called absolute stability [14]. To test for the stability, we will consider the test problem

$$
A_{r}+h B_{r}+h^{2} C_{r}+h^{3} D_{r}=\lambda y_{n}+h \lambda\left[b_{r 1}\left(A_{1}+h B_{1}+h^{2} C_{1}\right)+b_{r 2}\left(A_{2}+h B_{2}+h^{2} C_{2}\right)\right]
$$

Equating powers of $h$ and simplifying, we have

$$
\begin{gathered}
A_{r}=\lambda y_{n} \\
B_{r}=\lambda^{2} y_{n} \\
C_{r}=\lambda^{3} y_{n}\left(b_{r 1} a_{1}+b_{r 2} a_{2}\right) \\
D_{r}=\lambda^{4} y_{n}\left[\left(b_{r 1}\left(b_{11} a_{1}+b_{12} a_{2}\right)+b_{r 2}\left(b_{21} a_{1}+b_{22} a_{2}\right)\right]\right.
\end{gathered}
$$

Substituting (19)-(22) into (18), further into (3) and simplifying, then from (2) we have:

$$
\begin{aligned}
\frac{y_{n+1}}{y_{n}}=1 & +h \lambda+\frac{1}{2} h^{2} \lambda^{2}\left(a_{1}+a_{2}\right)+\frac{1}{6} h^{3} \lambda^{3}\left(3 a_{1}\left(b_{21}+b_{11}\right)+3 a_{2}\left(b_{22}+b_{12}\right)+a_{1} a_{2}\right) \\
& +\frac{1}{6} h^{4} \lambda^{4}\left(\left(b_{11} a_{1}+b_{12} a_{2}\right)\left(3 b_{21}+3 b_{11}+a_{2}\right)+\left(b_{21} a_{1}+b_{22} a_{2}\right)\left(3 b_{22}+3 b_{21}+a_{1}\right)\right) \\
& -\frac{1}{24} h^{3} \lambda^{3}\left(a_{1}+a_{2}\right)^{2}-\frac{1}{4} h^{4} \lambda^{4}\left(a_{1}+a_{2}\right)\left(a_{1}\left(b_{21}+b_{11}\right)+a_{2}\left(b_{22}+b_{12}\right)+a_{1} a_{2}\right)+\frac{1}{48} h^{4} \lambda^{4}\left(a_{1}+a_{2}\right)^{3}
\end{aligned}
$$

If we let $z=\lambda h$ and using the parameters in the solution of the sets of equations (i.e $a_{1}, a_{2}, b_{11}, b_{12}, b_{21}$ and $b_{22}$ ), we obtain the stability function to be

$$
R(z)=\frac{y_{n+1}}{y_{n}}=\frac{1+\frac{1}{2} z+\frac{1}{12} z^{2}}{1-\frac{1}{2} z+\frac{1}{12} z^{2}}
$$

This is a fourth-order rational approximation. Hence, it follows that our scheme has the same interval of absolute stability with the classical implicit Runge-Kutta proposed by Hammer and Hollingsworth in [9]. 


\subsection{Convergence of the Scheme}

From (23), we have

$$
\begin{aligned}
& y_{n+1}=y_{n}\left[1+h \lambda+\frac{1}{2} h^{2} \lambda^{2}\left(a_{1}+a_{2}\right)+\frac{1}{6} h^{3} \lambda^{3}\left(3 a_{1}\left(b_{21}+b_{11}\right)+3 a_{2}\left(b_{22}+b_{12}\right)+a_{1} a_{2}\right)\right. \\
& +\frac{1}{6} h^{4} \lambda^{4}\left(\left(b_{11} a_{1}+b_{12} a_{2}\right)\left(3 b_{21}+3 b_{11}+a_{2}\right)+\left(b_{21} a_{1}+b_{22} a_{2}\right)\left(3 b_{22}+3 b_{21}+a_{1}\right)\right) \\
& \left.-\frac{1}{24} h^{3} \lambda^{3}\left(a_{1}+a_{2}\right)^{2}-\frac{1}{4} h^{4} \lambda^{4}\left(a_{1}+a_{2}\right)\left(a_{1}\left(b_{21}+b_{11}\right)+a_{2}\left(b_{22}+b_{12}\right)+a_{1} a_{2}\right)+\frac{1}{48} h^{4} \lambda^{4}\left(a_{1}+a_{2}\right)^{3}\right]
\end{aligned}
$$

Applying the initial condition $\mathrm{y}(0)=1$ and keep $x_{n}$

fixed as $h \rightarrow 0$

$$
\begin{aligned}
& y_{n}= {\left[h \lambda+\frac{1}{2} h^{2} \lambda^{2}\left(a_{1}+a_{2}\right)+\frac{1}{6} h^{3} \lambda^{3}\left(3 a_{1}\left(b_{21}+b_{11}\right)+3 a_{2}\left(b_{22}+b_{12}\right)+a_{1} a_{2}\right)\right.} \\
&+\frac{1}{6} h^{4} \lambda^{4}\left(\left(b_{11} a_{1}+b_{12} a_{2}\right)\left(3 b_{21}+3 b_{11}+a_{2}\right)+\left(b_{21} a_{1}+b_{22} a_{2}\right)\left(3 b_{22}+3 b_{21}+a_{1}\right)\right) \\
&\left.-\frac{1}{24} h^{3} \lambda^{3}\left(a_{1}+a_{2}\right)^{2}-\frac{1}{4} h^{4} \lambda^{4}\left(a_{1}+a_{2}\right)\left(a_{1}\left(b_{21}+b_{11}\right)+a_{2}\left(b_{22}+b_{12}\right)+a_{1} a_{2}\right)+\frac{1}{48} h^{4} \lambda^{4}\left(a_{1}+a_{2}\right)^{3}\right]^{\frac{x_{n}}{h}} \\
& \ln y_{n}=\frac{x_{n}}{h} \ln \left[h \lambda+\frac{1}{2} h^{2} \lambda^{2}\left(a_{1}+a_{2}\right)+\frac{1}{6} h^{3} \lambda^{3}\left(3 a_{1}\left(b_{21}+b_{11}\right)+3 a_{2}\left(b_{22}+b_{12}\right)+a_{1} a_{2}\right)\right. \\
& \quad+\frac{1}{6} h^{4} \lambda^{4}\left(\left(b_{11} a_{1}+b_{12} a_{2}\right)\left(3 b_{21}+3 b_{11}+a_{2}\right)+\left(b_{21} a_{1}+b_{22} a_{2}\right)\left(3 b_{22}+3 b_{21}+a_{1}\right)\right) \\
&\left.\quad-\frac{1}{24} h^{3} \lambda^{3}\left(a_{1}+a_{2}\right)^{2}-\frac{1}{4} h^{4} \lambda^{4}\left(a_{1}+a_{2}\right)\left(a_{1}\left(b_{21}+b_{11}\right)+a_{2}\left(b_{22}+b_{12}\right)+a_{1} a_{2}\right)+\frac{1}{48} h^{4} \lambda^{4}\left(a_{1}+a_{2}\right)^{3}\right]
\end{aligned}
$$

Therefore

$$
\begin{aligned}
\lim _{h \rightarrow 0} \ln y_{n}= & x_{n} \lim _{h \rightarrow 0} \frac{1}{h} \ln \left[h \lambda+\frac{1}{2} h^{2} \lambda^{2}\left(a_{1}+a_{2}\right)+\frac{1}{6} h^{3} \lambda^{3}\left(3 a_{1}\left(b_{21}+b_{11}\right)+3 a_{2}\left(b_{22}+b_{12}\right)+a_{1} a_{2}\right)\right. \\
& \left.+\frac{1}{6} h^{4} \lambda^{4}\left(\left(b_{11} a_{1}+b_{12} a_{2}\right)\left(3 b_{21}+3 b_{11}+a_{2}\right)+\left(b_{21} a_{1}+b_{22} a_{2}\right)\left(3 b_{22}+3 b_{21}+a_{1}\right)\right)\right) \\
& \left.-\frac{1}{24} h^{3} \lambda^{3}\left(a_{1}+a_{2}\right)^{2}-\frac{1}{4} h^{4} \lambda^{4}\left(a_{1}+a_{2}\right)\left(a_{1}\left(b_{21}+b_{11}\right)+a_{2}\left(b_{22}+b_{12}\right)+a_{1} a_{2}\right)+\frac{1}{48} h^{4} \lambda^{4}\left(a_{1}+a_{2}\right)^{3}\right]
\end{aligned}
$$

By l'Hopital's rule we obtain,

$$
\lim _{h \rightarrow 0} \ln y_{n}=x_{n} \lambda
$$

Hence,

$$
\lim _{h \rightarrow 0} y_{n}=e^{x_{n} \lambda}
$$

Thus, the method is consistent and convergent. This concludes the proof.

\section{Numerical Experiment}

Our aim in this section is to see how well our method compares with other methods when solving non-stiff and stiff problems. We compared the new method with classical implicit Runge-Kutta (IRK4) and 2-stage third order diagonal Runge-Kutta (DIRK3) methods.
Example 1:

Consider the non-stiff initial problem:

$$
y^{\prime}(x)=1+y^{2}, \quad y(0)=1
$$

with the theoretical solution $y(x)=\tan \left(x+\frac{\pi}{4}\right)$. The computation were carried out using step size $\mathrm{h}=0.01$. The numerical result of the problem is represented in figure 1.

Example 2:

Consider the stiff initial problem:

$$
y^{\prime}=-15 y(x), \quad y(0)=1
$$

with the theoretical solution $y(x)=e^{-15 x}$ with $\mathrm{y}(\mathrm{x}) \rightarrow 0$ The computation were carried out using step size $h=0.01$. The numerical result of the problem is represented in the figure 2 .

Table 1. Numerical Results of Problem 1\&2.

\begin{tabular}{llllll}
\hline Problem 1 & & Problem 2 & Method & Absolute Error \\
\hline h & Method & Absolute Error & h & DIRK3 & $1.419593 \mathrm{E}-6$ \\
0.01 & DIRK3 & $3.498591 \mathrm{E}-8$ & & IRK4 & $5.748734 \mathrm{E}-8$ \\
& IRK4 & $4.178201 \mathrm{E}-9$ & 0.01 & HIRK & $6.683543 \mathrm{E}-9$ \\
& HIRK & $1.144002 \mathrm{E}-9$ & & DIRK3 & $3.498591 \mathrm{E}-6$ \\
0.02 & DIRK3 & $6.689592 \mathrm{E}-8$ & & IRK4 & $1.376677 \mathrm{E}-7$ \\
\hline
\end{tabular}




\begin{tabular}{llllll}
\hline Problem 1 & & \multicolumn{3}{l}{ Problem 2 } \\
\hline $\mathbf{h}$ & Method & Absolute Error & h & Method & Absolute Error \\
\hline \multirow{4}{*}{0.03} & HIRK & $1.443198 \mathrm{E}-9$ & & HIRK & $1.559419 \mathrm{E}-8$ \\
& DIRK3 & $1.168344 \mathrm{E}-7$ & & DIRK3 & $6.689591 \mathrm{E}-6$ \\
& IRK4 & $1.381108 \mathrm{E}-8$ & 0.03 & IRK4 & $2.684741 \mathrm{E}-7$ \\
0.04 & HIRK & $7.611098 \mathrm{E}-10$ & & HIRK & $2.912114 \mathrm{E}-8$ \\
& DIRK3 & $1.849974 \mathrm{E}-7$ & & DIRK3 & $1.168344 \mathrm{E}-5$ \\
& IRK4 & $1.988415 \mathrm{E}-8$ & 0.04 & IRK4 & $5.276223 \mathrm{E}-7$ \\
0.05 & HIRK & $1.702371 \mathrm{E}-9$ & & HIRK & $5.260237 \mathrm{E}-8$ \\
& DIRK3 & $1.972515 \mathrm{E}-7$ & & DIRK3 & $1.849972 \mathrm{E}-5$ \\
& IRK4 & $2.556197 \mathrm{E}-8$ & 0.05 & IRK4 & $1.201972 \mathrm{E}-6$ \\
\end{tabular}
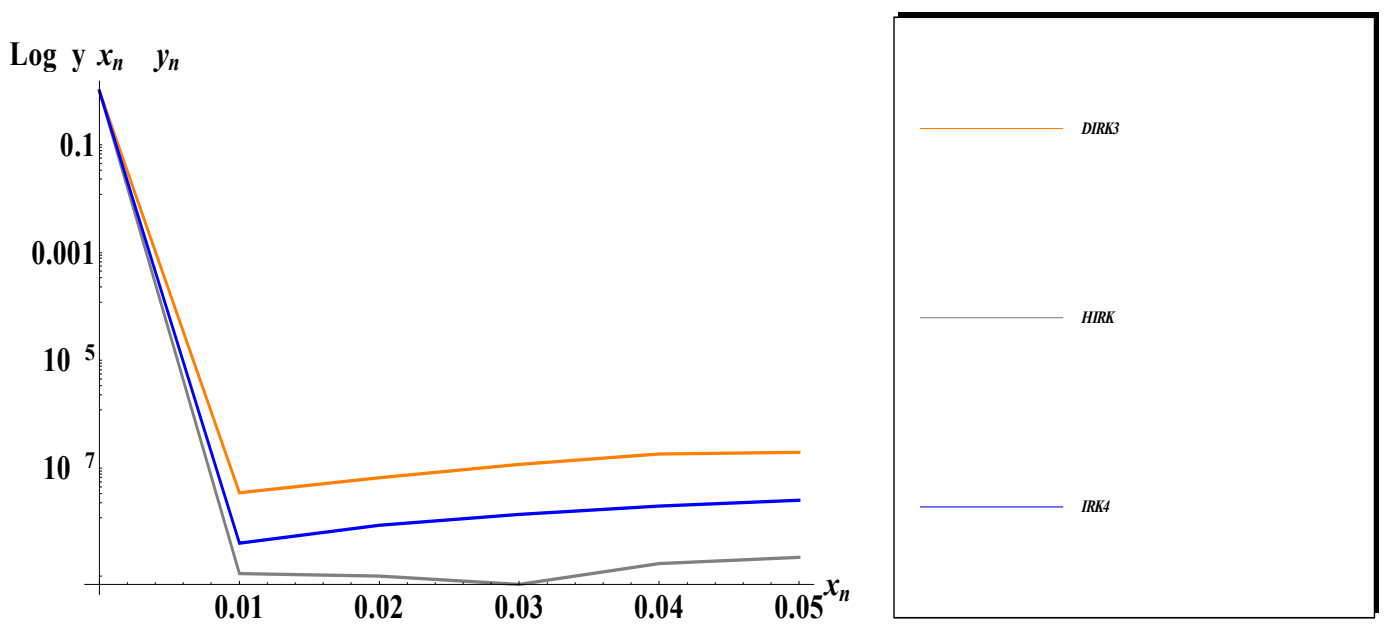

Figure 1. The Graph of DIRK3, IRK4 and HIRK for Problem 1.

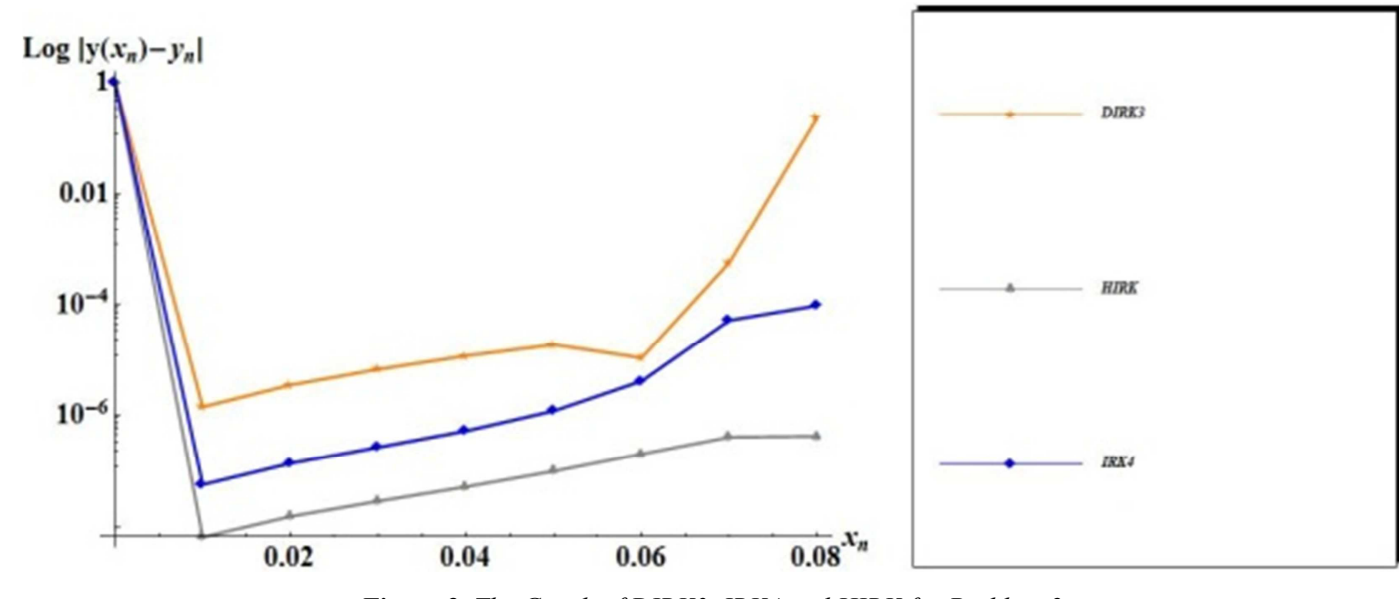

Figure 2. The Graph of DIRK3, IRK4 and HIRK for Problem 2.

Remark 4.2: From Table 1, Figure 1 and Figure 2 we observed that our method have better accuracy than Diagonal Implicit Runge-Kutta Method of order 3 (DIRK3) and classical Implicit Runge-Kutta Method of order 4 (IRK4).

\section{Conclusion}

The increasing popularity of the applicability of other types of mean in the derivation of explicit Runge-Kutta methods motivated us to extend the idea to implicit Runge-Kutta method. In this paper, we constructed a new implicit Runge-Kutta method using heronian mean as a basis in the derivation. The scheme's qualitative properties were also established. From the results obtained via the numerical experiment, we confirmed that our scheme which was tagged HIRK method outperforms the existing methods such as diagonal implicit Runge-Kutta method of order 3 and the classical implicit Runge-Kutta method of order 4 when used to solve initial value problems in ordinary differential equations. Following the experimental study, we have shown that the use of other types of mean in the derivation of explicit Runge-Kutta methods can be extended to implicit Runge-Kutta methods; hence, opening a window of exploration for other types of mean in the derivation of 
implicit Runge-Kutta methods.

\section{References}

[1] Ababneh O. Y. and Ahmad R. "Construction of Third-Order Diagonal Implicit Runge-Kutta method for Stiff Problems". Chinese Physics Letters, Vol. 26, No. 8, Article ID 080503, 2009.

[2] Ademiluyi R. A., Babatola P. O. and Kayode S. J. "A new class of implicit Rational Runge-Kutta method for integration of stiff ODEs". Journal of Nigerian Mathematical Society, 21, (2002), 27-41.

[3] Ademiluyi R. A., Babatola P. O. and Kayode S. J."Semi-implicit Rational Runge-Kutta formulas of approximation of stiff initial value problems in ODEs". Journal of Nigerian Mathematical Science and Education, (2001), 11 25.

[4] Agam, S. A. and Yahaya, Y. A. (2014). A highly efficient implicit Runge-Kutta method for first order ordinary differential equations. African Journal of Mathematics and Computer Science Research, 7 (5), 55-60.

[5] Akanbi M. A. (2011). "On 3-Stage Geometric Explicit Runge-Kutta Methods for Singular Initial value Problems in Ordinary Differential equations". Computing, Springer Journals, Vol. 92, No. 3, 243263.

[6] Akanbi, M, A. and Okunuga, S. A. (2008). "On the Convergence and Stability of 2stage Multiderivative Explicit Runge-Kutta Methods". Journal of Nigerian Mathematical Society, Vol. 27.123143.

[7] Butcher, J. C. (2000). Numerical methods for ordinary differential equations in the 20th century. Journal of Computational and Applied Mathematics, 125 (1-2), 1-29.

[8] Chen D. J. L., "The Efficiency of Singly-Implicit Runge-Kutta Methods for Stiff Diffential Equations". Numerical Algorithm, Vol. 65, No. 3, pp. 533-554, 2014.

[9] Dahlquist G. (1956)."Convergence and Stability in the Numerical Integration of ODEs". Math. Scand. 4: 33353.

[10] Evans D. J. and Yaacob N. B. (1995). "A Fourth Order
Runge-Kutta Method Based on the Heronian Mean Formula”. International Journal of Computer Mathematics, 58, 1033115.

[11] Evans D. J. and Yaacob N. B. (1995).”A New Fourth Order Runge-Kutta Method Based on the Contraharmonic Mean". International Journal of Computer Mathematics, 57, 2499256.

[12] Jameson A. "Evaluation of Fully Implicit Runge-Kutta Schemes for Unsteady Flow Calculations". Journal of Scientific Computing, Vol. 73, No. 2-3, pp. 819-852, 2017.

[13] Jawias N. I. C., Ismail F., Suleiman M. and Jaafar A."Fourth Order Four-Stage Diagonally Implicit Runge-Kutta method for linear Ordinary Differential Equations". Malaysian Journal of Mathematical Sciences, vol. 4, pp. 955 105, 2010.

[14] Lambert J. D. (1991)."Numerical Methods for Ordinary Differential Systems". John Wiley and sons, England.

[15] Liao W. and Yan Y., "Singly Diagonally Implicit Runge-Kutta Method for Time-Dependent Reaction-Diffusion equation". Numerical Methods for Partial Differential equations, Vol. 27, No. 6, pp. 1423-1441, 2011.

[16] Liu M. Y., Zhang L. and Zhang C. F. "Study on Banded Implicit Runge-Kutta for Solving Stiff Differential Equations". Hindawi Mathematical Problems in Engineering, Volume 2019, Article ID 4850872, 8 Pages.

[17] Ponalagusamy P. J. A. and Chandra M. (2011), "Development of New Fifth-Order Fifth Stage Runge-Kutta Method on Heronian Mean". International Journal of Engineering Science for Advance Computing, Vol. 2, pp. 1622197.

[18] Wusu A. S., Akanbi M. A. and Bakre O. F. (2015). "On the Derivation and Implementation of a Four-Stage HarmonicExplicit Runge-Kutta Method”. Applied Mathematics, 2015, 6, 6944699.

[19] Wusu A. S., Okunuga S. A. and Sofoluwe A. B. (2012). “A Third-Order Harmonic Explicit Runge-Kutta Method for Autonomous Initial Value Problems". Global Journal of Pure and Applied Mathematics, 8, 4411451.

[20] Yaacob N. B. and Sanugi B. (1995). "A New Fourth Order Embedded Method Based on the Harmonic Mean". Mathematika, 14, 116. 